\title{
Direct imaging of antiferromagnetic domains and anomalous layer-dependent mirror symmetry breaking in atomically thin $\mathrm{MnPS}_{3}$
}

\author{
Zhuoliang Ni, ${ }^{1}$ Huiqin Zhang, ${ }^{2}$ David Hopper, ${ }^{1,2}$ Amanda V. Haglund, ${ }^{3}$ Nan Huang, ${ }^{3}$ Deep Jariwala, ${ }^{2}$ \\ Lee Bassett, ${ }^{2}$ David G. Mandrus, ${ }^{3,4}$ Eugene J. Mele, ${ }^{1}$ Charles L. Kane, ${ }^{1}$ and Liang $\mathrm{Wu}^{1}{ }^{1} *$ \\ ${ }^{1}$ Department of Physics and Astronomy, University of Pennsylvania, Philadelphia, Pennsylvania 19104, U.S.A \\ ${ }^{2}$ Department of Electrical and System Engineering, \\ University of Pennsylvania, Philadelphia, Pennsylvania 19104, U.S.A \\ ${ }^{3}$ Department of Materials Science and Engineering, \\ University of Tennessee, Knoxville, TN 37996, U.S.A. \\ ${ }^{4}$ Materials Science and Technology Division, Oak Ridge National Laboratory, Oak Ridge, TN, 37831, U.S.A.
}

(Dated: October 7, 2021)

\begin{abstract}
We have developed a sensitive cryogenic second-harmonic generation microscopy to study a van der Waals antiferromagnet $\mathrm{MnPS}_{3}$. We find that long-range Néel antiferromagnetic order develops from the bulk crystal down to the bilayer, while it is absent in the monolayer. Before entering the long-range antiferromagnetic ordered phase in all samples, an upturn of the second harmonic generation below $200 \mathrm{~K}$ indicates the formation of the short-range order and magneto-elastic coupling. We also directly image the two antiphase $\left(180^{\circ}\right)$ antiferromagnetic domains and thermally-induced domain switching down to bilayer. An anomalous mirror symmetry breaking shows up in samples thinner than ten layers for the temperature both above and below the Néel temperature, which indicates a structural change in few-layer samples. Minimal change of the second harmonic generation polar patterns in strain tuning experiments indicate that the symmetry crossover at ten layers is most likely an intrinsic property of $\mathrm{MnPS}_{3}$ instead of an extrinsic origin of substrate-induced strain. Our results show that second harmonic generation microscopy is a direct tool for studying antiferromagnetic domains in atomically thin materials, and opens a new way to study two-dimensional antiferromagnets.
\end{abstract}

Compared to ferromagnetic materials, antiferromagnetic (AFM) materials usually have a much higher magnon frequency in the terahertz regime and a more stable ground state to perturbations of external magnetic fields, making them promising platforms for spintronic devices operating in the terahertz frequency range. Twodimensional (2D) AFM materials are of particular interest recently [1-15], with the prospect of devices down to the atomically-thin limit. Contrary to the rapid development in 2D ferromagnetic materials [16-28], 2D AFM materials have been less explored due to the lack of direct probes on the AFM orders until very recently $[9,12-$ 14, 29]. Raman scattering [1, 2, 4, 5, 30, 31], magnetic tunneling [8] and magnon transport measurement reveal photons or the bandgap coupled to the AFM orders [7], but they do not directly probe the AFM order and the domains [25].

Commonly known as a probe of structural and surface inversion symmetry $(\mathcal{P})$ breaking, second harmonic generation (SHG) has also been demonstrated to be a direct probe to the long-range AFM orders and domains [13, 28, 29, 32, 33]. SHG can be classified into two kinds: i-type and c-type[32, 33]. The i-type SHG is invariant under the time-reversal symmetry $(\mathcal{T})$, and includes the electric-dipole (ED) SHG in noncentrosymmetric crystals and electric-quadruple (EQ) SHG. The EQ contribution does not require the condition of broken inversion symmetry in the lattice. The c-type SHG changes sign under the time-reversal transformation, and is only present if the spin structure breaks the inversion symmetry in centrosymmetric lattice. The c-type SHG has been very rare, and was observed in parity-time-reversal $(\mathcal{P} \mathcal{T})$ symmetric antiferromagnets such as $\mathrm{Cr}_{2} \mathrm{O}_{3}[32,33]$, bilayer $\mathrm{CrI}_{3}[28]$ and monolayer $\mathrm{MnPSe}_{3}[13]$. Below the transition temperature, the c-type SHG susceptibility is proportional to the order parameter [34]. The interference between the c-type and i-type SHG has been used to directly image AFM domains [13, 32, 33]. Nevertheless, studies on imaging of AFM domains in atomically thin crystals have been very limited [13, 28].

The AFM transition metal thiophosphates $\mathrm{MPS}_{3}$ $(\mathrm{M}=\mathrm{Mn}, \mathrm{Fe}, \mathrm{Ni})$ have attracted lots of interests recently including the many-body exciton in $\mathrm{NiPS}_{3}[9,12,14]$, Ising antiferromagntism in $\mathrm{FePS}_{3}[1,2,30,35]$, and magneto-electric effect in $\mathrm{MnPS}_{3}$ [36]. Also, they order in different spin structures despite the same monoclinic structure in space group No. $12 . \mathrm{NiPS}_{3}$ and $\mathrm{FePS}_{3}$ have zigzag orders, but $\mathrm{MnPS}_{3}$ has the Néel order. Among them, $\mathrm{MnPS}_{3}$ breaks $\mathcal{P}$ and $\mathcal{T}$ below the Néel temperature $\left(T_{N}\right)$, but respects $\mathcal{P} \mathcal{T}$ symmetry, and, therefore, it allows the c-type ED SHG in the AFM phase. A previous SHG study on $\mathrm{MnPS}_{3}$ demonstrated a phase-transitionlike feature around $T_{N}$ down to seven-layer $(7 \mathrm{~L})$, but did not observe short-range order above $T_{N}$, Néel-type AFM domains, and did not provide a clear evidence for the c-type SHG that changes sign under time-reversal transformation [29]. Very high laser power was used in the previous study [29], because of the material's weak second-order response. Note that the SHG intensity from $\mathrm{MnPS}_{3}$ is at least two orders of magnitude lower than 
(a)

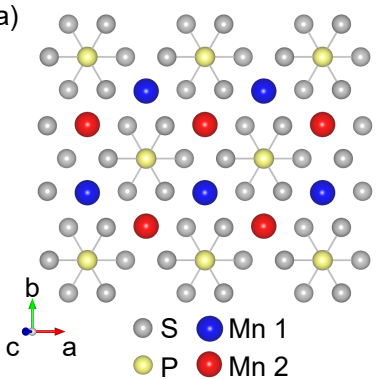

(c)

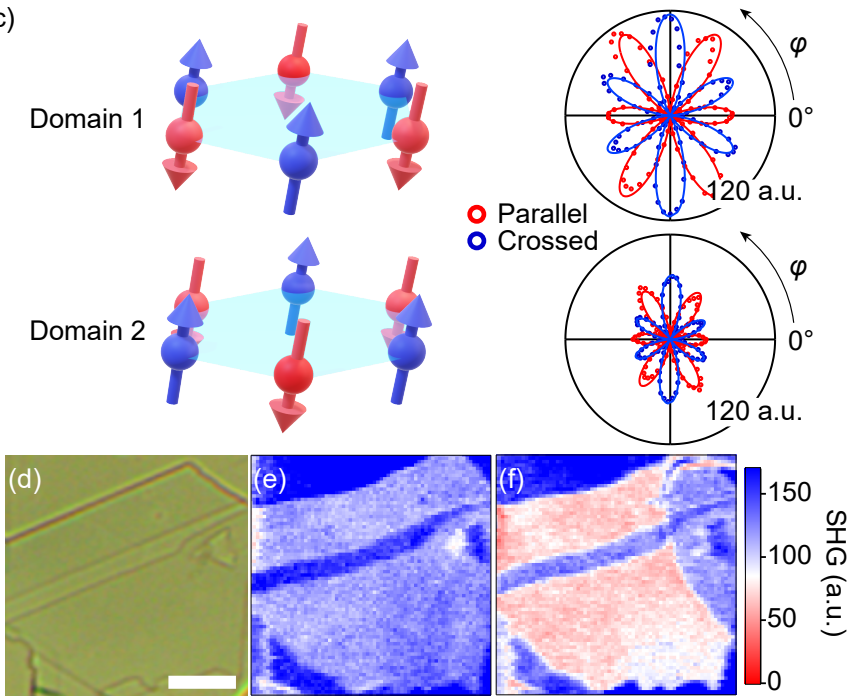

FIG. 1. (a) Top view of the monolayer crystal structure of $\mathrm{MnPS}_{3}$. (b) Temperature dependence of SHG intensity of two domains in the same $\mathrm{MnPS}_{3}$ bulk crystal. The inset shows SHG intensity near the transition temperature. (c) Two possible spin configurations of $\mathrm{Mn}$ atoms in a singlelayer. Red arrows indicate spin directions of Mn atoms. The SHG polar patterns corresponding to two domains are shown on the right. The measured data are shown in dots and best fits are shown in solid lines. (d)-(f) Optical image and SHG intensity images at $5 \mathrm{~K}$ of a bulk $\mathrm{MnPS}_{3}$ sample. Scale bar: $50 \mu \mathrm{m}$.

that in $\mathrm{MnPSe}_{3}$ due to its much weaker spin-orbital coupling [13]. Fast sample degradation due to high laser power happened within hours, which prevent a systematic study of the layer-dependent antiferromagnetism, domains, short-range order and time-reversal-odd c-type SHG in $\mathrm{MnPS}_{3}$ [29].

In this work, we develop a sensitive cryogenic scanning SHG microscopy with the photon detection sensitivity of 0.1 counts per second (c.p.s.) by using a photon counter to systematically study the layer-dependent antiferromagnetism in $\mathrm{MnPS}_{3}$ down to the monolayer. We observe the sign change of the c-type SHG coefficient below $T_{N}$, and two antiphase $\left(180^{\circ}\right) \mathrm{AFM}$ domains with spins reversed by time-reversal transformation. The long-range AFM order, evident from the c-type SHG, is present from the bulk to bilayer, but is absent in the monolayer. Between $T_{N}$ and $200 \mathrm{~K}$, we observe an in- crease of the SHG as a function of decreasing temperature, which indicates correlation-induced short-range order and the magneto-elastic coupling. Polarized SHG measurement shows the mirror-symmetry breaking below $10 \mathrm{~L}$ both below and above $T_{N}$, indicating a symmetry crossover in the structure in thin flakes.

The bulk crystal of $\mathrm{MnPS}_{3}$ belongs to the point group of $2 / \mathrm{m}$ above $T_{N} \approx 78 \mathrm{~K}$ and magnetic point group $2^{\prime} / \mathrm{m}$ below $T_{N}$. Neutron scattering measurements indicate its magnetic system to be Heisenberg type with tiny dipolar anisotropy and single-ion anisotropy [36-40]. The lattice structure is shown in Fig. 1(a). In the single layer, Mn atoms form a honeycomb lattice in the $a b$ plane, and the two $\mathrm{Mn}$ atoms with opposite spin directions are marked by different colors, showing its AFM Néel order in a single layer. The spins are mainly out of plane, but 8 degree from the surface normal direction. The honeycomb layers stack along the $c$ axis and with a shift of $1 / 3$ of the lattice constant along the $a$ axis, which gives rise to a centrosymmetric lattice. There is a mirror symmetry perpendicular to the $b$ axis. Interestingly, the formation of Néel AFM order breaks the inversion symmetry, and therefore it allows c-type ED SHG.

We first explore the SHG signal in a bulk crystal with thickness around $10 \mu \mathrm{m}$ as shown in Fig. 1(d). Under normal incidence, we send in laser pulses centered at 800 $\mathrm{nm}$ (with $80 \mathrm{MHz}$ repetition rate and around $50 \mathrm{fs}$ duration) and measure SHG at $400 \mathrm{~nm}[13,41]$. We focus $5 \mathrm{~mW}$ on a spot with a diameter of $10 \mu \mathrm{m}$. By consecutively doing thermal cycles at one spot, Fig. 1(b) shows that there are only two SHG traces when crystal is cooled from the paramagnetic to the AFM states[42]. At high temperatures from $200 \mathrm{~K}$ to $300 \mathrm{~K}$, the temperatureindependent SHG signal contributes from the electricquadruple (EQ) term of the lattice. From $200 \mathrm{~K}$ to 78 $\mathrm{K}\left(T_{N}\right)$, the two curves still overlap and rise gradually. The slow rising of EQ term in SHG is believed to originate from short-range orders of spins due to correlationinduced lattice distortions [38], which is also known as magneto-elastic coupling [11]. A similar phenomenon was observed previously in a van-der Waals ferromagnet [48]. The two curves split at $77.7( \pm 0.3) \mathrm{K}$, which we define as the onset of c-type ED SHG and therefore the $T_{N}$. To explain the transition behavior, we write the interfering SHG intensity from ED and EQ terms as

$$
I_{i}^{S H G}=\left( \pm \chi_{i j k}^{E D}\left(T \leq T_{N}\right) E_{j} E_{k}+\chi_{i j k l}^{E Q}(T) E_{j} \nabla_{k} E_{l}\right)^{2},
$$

where $\chi_{i j k}^{E D}(T) \propto M \propto\left(T_{N}-T\right)^{\beta}$ is the ED SHG coefficient [34]. ( $M$ is the staggered magnetization of the two Mn sites and is the order parameter.) The \pm sign corresponding to two $\mathcal{P} \mathcal{T}$-related domains as shown in $1(\mathrm{c}) . \chi^{E Q}(T)$ is present at all temperature and is identical in both domains, since magneto-elastic distortions caused by the two domains are the same. The splitting of SHG intensity of the two domains at $T_{N}$ is the direct 
evidence of the time-reversal-odd c-type ED SHG and therefore the long-range AFM order in $\mathrm{MnPS}_{3}$. As observed in a centrosymmetric 2D ferromagnet [48] and the $2 \mathrm{D}$ zigzag antiferromagnet $\mathrm{NiPS}_{3}[42]$, just observing the rising of SHG across $T_{N}$ without observing a sign change could contribute from i-type SHG due to magneto-elastic coupling and might not be proportional to the order parameter. Note that the inset figure in Fig. 1(b) shows a change of slope in the curve of domain 1 around $76 \mathrm{~K}$, which might be related with the crossover of $3 \mathrm{D}$ to $2 \mathrm{D}$ critical behaviour [38, 42].

In Fig. 1(e,f), we show SHG intensity mapping at $5 \mathrm{~K}$ after two consecutive thermal cycles in the area marked by the optical image in Fig. 1(d). Fig. 1(e) shows a single domain, while Fig. 1(f) shows two domains. To the best of our knowledge, this is the first report of direct observation of $\mathrm{AFM}$ domains in $\mathrm{MnPS}_{3}$. We further measure the SHG polar patterns in these two domains as shown on the right of Fig. 1(b). Here "parallel" and "crossed" refer to configurations of the polarization of the incident and SHG laser pulses[13, 41], and they have different angle dependence on $\chi_{i j k}^{E D}$, which reveal the symmetry of the material (see supplementary note $\mathrm{S} 1$ ). The $\phi=0$ direction in parallel configuration corresponds to the $a$-axis of the $\mathrm{MnPS}_{3}$ crystal. These patterns can be well fitted (solid lines) by the symmetry analysis based on the material's space group. The SHG maps in Fig. 1(e,f) and traces in Fig. 1(b) are taken when $\phi=0^{\circ}$ in the parallel configuration. We fix the laser position at one spot, and confirm that there are only two kinds of polar patterns after ten thermal cycles. The two domains switch to each other by the time-reversal transformation. As a result, the c-type ED SHG is also called non-reciprocal SHG. The polar patterns also confirm that the mirror plane perpendicular to the $b$ axis and the tilting angle of spins are in the $a c$ plane.

Next, we study whether the long-range order and domains sustain in the atomically thin limit. We use a relatively low power of $2 \mathrm{~mW}$ over the beam diameter of $10 \mu \mathrm{m}$ to avoid damage to atomically-thin samples. Note that our incident power per area is around 50 times lower than that of the previous study [29]. The optical image of a large nine-layer (9L) sample on $\mathrm{SiO}_{2}(90 \mathrm{~nm}) / \mathrm{Si}(0.5$ $\mathrm{mm}$ ) is shown Fig. 2(a), and the thickness is determined by a combination of atomic force microscopy measurement and color contrast measurement[42]. We perform a SHG mapping at $5 \mathrm{~K}$ (Fig. 2(b)) with the polarization at $172^{\circ}$ peak position in the parallel configuration of domain 1 in Fig. 2(e). Two AFM domains are observed represented by the darker (domain 2) and the brighter (domain 1) areas. Next, we warm up the sample above $T_{N}$ and cool it back to $5 \mathrm{~K}$, and the new SHG mapping is shown as Fig. 2(c). The different distribution of two domains indicates that Néel vectors switch freely between two directions across $T_{N}$, in consistency with the bulk. Fig. 2(d) shows the temperature-dependent intensity of
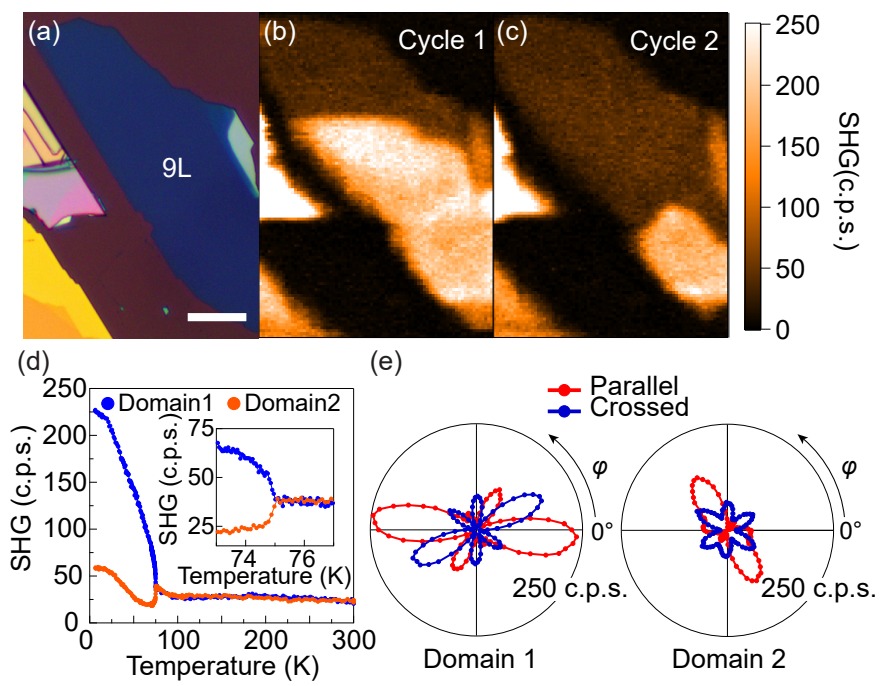

FIG. 2. (a) Optical image of the $9 \mathrm{~L} \mathrm{MnPS}_{3}$ sample. Scale bar: $20 \mu \mathrm{m}$. (b-c) SHG intensity mapping measured at 5 K. (d) Temperature dependence of SHG intensity of two domains Inset shows a closer look near transition temperature. (e) Polarization dependence of SHG intensity of two domains measured at $5 \mathrm{~K}$.

two domains with $\phi=172^{\circ}$ in the parallel configuration. We also perform over nine thermal cycles and confirm that there are only two domains[42]. Surprisingly, both polar patterns in Fig. 2(e) show the breaking of mirror symmetry in the spin structure of this $9 \mathrm{~L}$ sample.

To approach the 2D limit, we exfoliate $\mathrm{MnPS}_{3}$ samples from six-layer to monolayer. The isolated samples with diameters at least three times larger than laser spot are chosen. The experimental results are summarized in Fig. 3. Polar patterns of the two domains at $5 \mathrm{~K}$ in $2,3,5,6$ layer samples are shown in Fig. 3(a). Similar to the 9L sample, the mirror symmetry is broken in these samples. The temperature-dependent SHG intensity of these samples is shown in Fig. 3(b). The layer-dependent intensity at $5 \mathrm{~K}$ (chosen at the maximum in the parallel pattern) is shown in Fig. 3(c). As each $\mathrm{MnPS}_{3}$ layer produces in-phase SHG response, the intensity of samples from $3 \mathrm{~L}$ to $22 \mathrm{~L}$ nearly follows the dependence of the square of the number of layers, as observed in other inversion-breaking $2 \mathrm{D}$ materials $[13,49]$. There is a strong suppression of SHG intensity in the bilayer samples. We confirm that the suppression is not due to degration in air as the SHG intensity of a $2 \mathrm{~L}$ sample exfoliated in a glove box is the same[42]. We measure three monolayer samples exfoliated in air and one monolayer sample exfoliated in the glove box, but we are unable to detect a temperaturedependent SHG signal within our setup sensitivity of 0.1 c.p.s.[42]. Therefore, we think the absence of long-range order in the monolayer is not due to air degration. The Néel temperature, which we extract at the splitting of SHG of two domains, is plotted in Fig. 3(d). It is also suppressed with decreasing of the layer number(below 

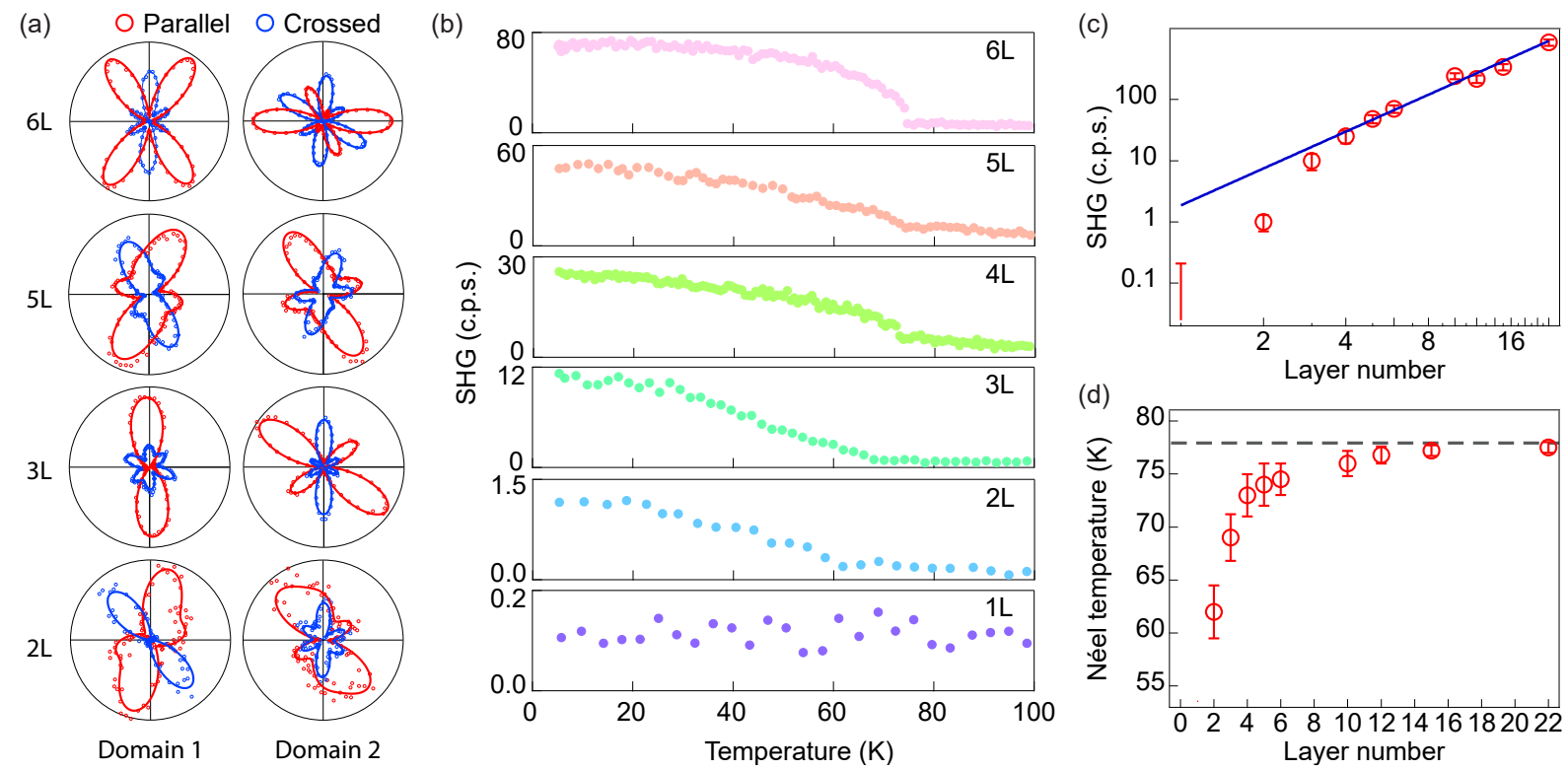

FIG. 3. (a) SHG polar patterns of two AF domains in different layers measured at 5 K. (b) Temperature dependence of SHG intensity at the crossed peak of domain 1 from different layers. (c) Layer number dependence of SHG intensity. (d) Layer number dependence of Néel temperature.

10L).

In order to study the origin of the mirror symmetry breaking, we systematically investigate the layer dependence. As shown in Fig. 4, there is a clear difference between the 12L and the 10L samples. For samples thicker than $12 \mathrm{~L}$, the SHG patterns show a mirror symmetry, and the six lobes are of comparable magnitude, which is similar to the bulk sample in Fig. 1. However, for those thinner than 10L, the mirror symmetry is absent in 10 $\mathrm{K}$, similar to the data shown in Fig. 2 and Fig. 3. Note that at $200 \mathrm{~K}$, where the EQ SHG signal is mostly from the lattice and is decoupled to the short-range spin correlation, the mirror symmetry is still absent. Therefore, the absence of the mirror symmetry below 10L has a lattice origin. A recently near-field infrared spectroscopy study on the phonon spectrum and density functional theory calculation on the same material also shows a crossover of the lattice symmetry around $10 \mathrm{~L}$, and they attribute it to the symmetry crossover from $2 / \mathrm{m}$ to a higher symmetry $\mathrm{P} 3{ }_{1} \mathrm{~m}$ with three mirrors $[50,51]$. In our experiment, though observing the similar thickness for the crossover, we find the symmetry of $\mathrm{MnPS}_{3}$ samples thinner than $10 \mathrm{~L}$ in the paramagnetic state is actually lower than thicker samples. We tend to believe that the mirror symmetry breaking is an intrinsic property of very thin $\mathrm{MnPS}_{3}$ crystal as the change of polar patterns between $12 \mathrm{~L}$ and $10 \mathrm{~L}$ is dramatic. We tend to think that mirror symmetry breaking is not due to stacking faults as it has a systematic thickness dependence. Also, to exclude the strain effect caused by the substrate, we perform the strain-tuning experiments on a $9 \mathrm{~L}$ and a $16 \mathrm{~L}$ samples. No strain-induced mirror symmetry breaking is observed in the 16L sample. Also, both samples do not show the change in the SHG polar pattern by the 5 $\%$ strain applied on the substrate[42]. Further detailed structure measurement and density functional theory calculation are called to reveal the origin of mirror symmetry breaking below 10L.

The long-range order in bulk $\mathrm{MnPS}_{3}$ is believed to result from the anisotropy and the weak interplanar coupling [37, 38]. The suppression of the Néel temperature possibly results from reduced interplanar coupling in atomically thin samples $[6,17]$. Nevertheless, consider the symmetry of the spin structure is different in samples less than 10L, it might not be valid to use the same bulk Hamiltonian to explain atomically thin samples. We also can not rule out the possibility of disorder effect on the absence of the ordering in the monolayer. More discussions about the suppression of long-range order in the monolayer can be found in Ref.[42]. We hope more future works will investigate the monolayer $\mathrm{MnPS}_{3}$.

To summarize, by using a sensitive SHG microscopy, we systematically study the layer dependence of AFM states in $\mathrm{MnPS}_{3}$, and observe the switching of Néel-type AFM domains. Looking forward, We believe that this work is critical to understanding the $2 \mathrm{D}$ antiferromagnetism in this compound, and application for $2 \mathrm{D}$ terahertz AFM spintronic devices.

We thank S.W. Cheong for helpful discussions. The project design, data collection and analysis are supported by L.W.'s startup package at the University of Pennsylvania. The development of the SHG photon counter is supported by the ARO YIP award under 


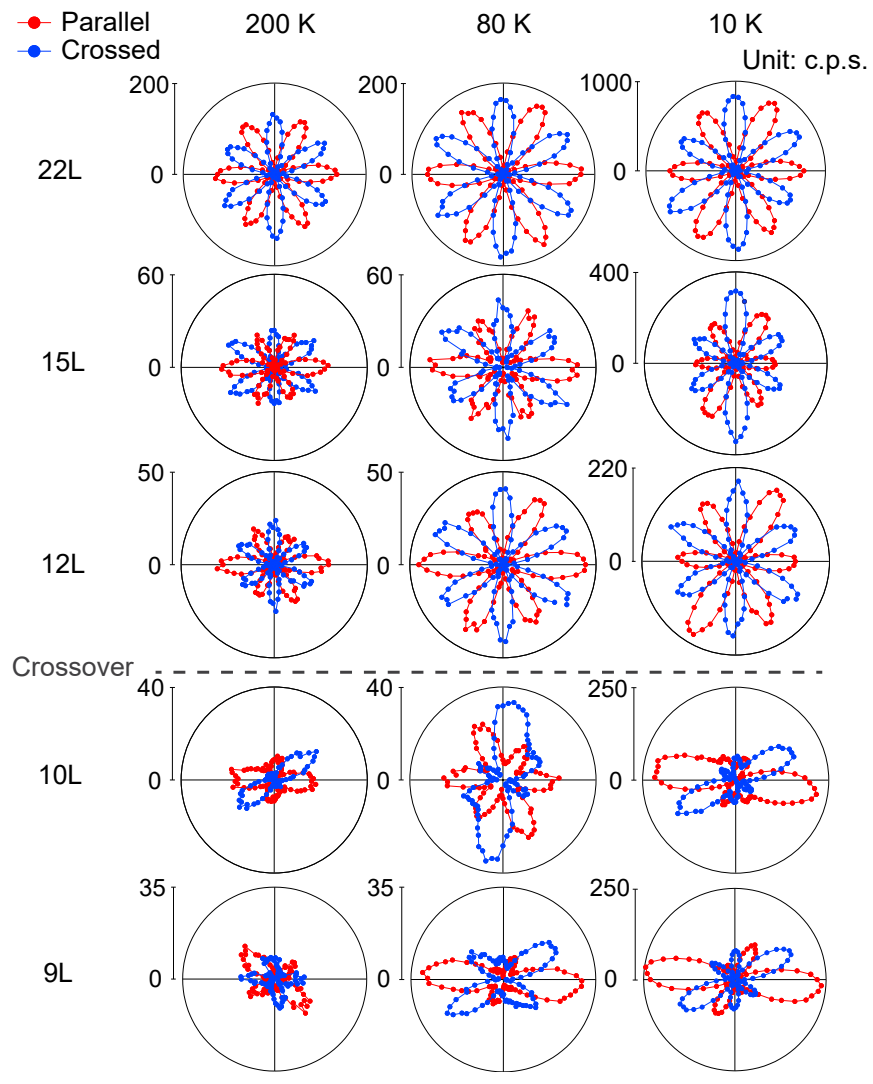

FIG. 4. Parallel (blue) and crossed (red) polar patterns of different layers. The dots with lines are the data.

the Grant W911NF1910342. The measurement by the atomic force microscopy is supported by the ARO MURI under the Grant W911NF2020166. Z.N., H.Z., D.J. and L.W. acknowledge partial support from National Science Foundation supported University of Pennsylvania Materials Research Science and Engineering Center (MRSEC)(DMR-1720530). E.J.M. acknowledges support from NSF EAGER 1838456. C.L.K is supported by a Simons Investigator grant from the Simons Foundation. D.G.M acknowledges support from the Gordon and Betty Moore Foundation's EPiQS Initiative, Grant GBMF9069. Z.N. and H.Z. also acknowledge support from Vagelos Institute of Energy Science and Technology graduate fellowship at the University of Pennsylvania.

* liangwu@sas.upenn.edu

[1] Jae-Ung Lee, Sungmin Lee, Ji Hoon Ryoo, Soonmin Kang, Tae Yun Kim, Pilkwang Kim, Cheol-Hwan Park, Je-Geun Park, and Hyeonsik Cheong, "Ising-type magnetic ordering in atomically thin $\mathrm{FePS}_{3}$," Nano Lett. 16, 7433-7438 (2016).

[2] Xingzhi Wang, Kezhao Du, Yu Yang Fredrik Liu, Peng $\mathrm{Hu}$, Jun Zhang, Qing Zhang, Man Hon Samuel Owen, Xin Lu, Chee Kwan Gan, and Pinaki Sengupta, "Raman spectroscopy of atomically thin two-dimensional magnetic iron phosphorus trisulfide $\left(\mathrm{FePS}_{3}\right)$ crystals," 2D Mater. 3, 031009 (2016).

[3] So Yeun Kim, Tae Yun Kim, Luke J. Sandilands, Soobin Sinn, Min-Cheol Lee, Jaeseok Son, Sungmin Lee, KiYoung Choi, Wondong Kim, Byeong-Gyu Park, C. Jeon, Hyeong-Do Kim, Cheol-Hwan Park, Je-Geun Park, S. J. Moon, and T. W. Noh, "Charge-spin correlation in van der waals antiferromagnet nips 3 ," Phys. Rev. Lett. 120, 136402 (2018).

[4] Yu-Jia Sun, Qing-Hai Tan, Xue-Lu Liu, Yuan-Fei Gao, and Jun Zhang, "Probing the Magnetic Ordering of Antiferromagnetic $\mathrm{MnPS}_{3}$ by Raman Spectroscopy," J. Phys. Chem 10, 3087-3093 (2019).

[5] Kangwon Kim, Soo Yeon Lim, Jungcheol Kim, Jae-Ung Lee, Sungmin Lee, Pilkwang Kim, Kisoo Park, Suhan Son, Cheol-Hwan Park, Je-Geun Park, and Hyeonsik Cheong, "Antiferromagnetic ordering in van der Waals 2D magnetic material $\mathrm{MnPS}_{3}$ probed by Raman spectroscopy," 2D Mater. 6, 041001 (2019).

[6] Kangwon Kim, Soo Yeon Lim, Jae-Ung Lee, Sungmin Lee, Tae Yun Kim, Kisoo Park, Gun Sang Jeon, CheolHwan Park, Je-Geun Park, and Hyeonsik Cheong, "Suppression of magnetic ordering in XXZ-type antiferromagnetic monolayer $\mathrm{NiPS}_{3}$," Nat. Commun. 10, 345 (2019).

[7] Wenyu Xing, Luyi Qiu, Xirui Wang, Yunyan Yao, Yang Ma, Ranran Cai, Shuang Jia, X. C. Xie, and Wei Han, "Magnon Transport in Quasi-Two-Dimensional van der Waals Antiferromagnets," Phys. Rev. X 9, 011026 (2019).

[8] Gen Long, Hugo Henck, Marco Gibertini, Dumitru Dumcenco, Zhe Wang, Takashi Taniguchi, Kenji Watanabe, Enrico Giannini, and Alberto F. Morpurgo, "Persistence of magnetism in atomically thin $\mathrm{MnPS}_{3}$ crystals," Nano Lett. 20, 2452-2459 (2020).

[9] Soonmin Kang, Kangwon Kim, Beom Hyun Kim, Jonghyeon Kim, Kyung Ik Sim, Jae-Ung Lee, Sungmin Lee, Kisoo Park, Seokhwan Yun, Taehun Kim, Abhishek Nag, Andrew Walters, Mirian Garcia-Fernandez, Jiemin Li, Laurent Chapon, Ke-jin Zhou, Young-Woo Son, JaeHoon Kim, Hyeonsik Cheong, and Je-Geun Park, "Coherent many-body exciton in van der Waals antiferromagnet $\mathrm{NiPS}_{3}$," Nature 583, 785-789 (2020).

[10] Makars Šiškins, Martin Lee, Samuel Mañas-Valero, Eugenio Coronado, Yaroslav M Blanter, Herre SJ van der Zant, and Peter G Steeneken, "Magnetic and electronic phase transitions probed by nanomechanical resonators," Nat. Commun. 11, 2698 (2020).

[11] Diana Vaclavkova, Alex Delhomme, Clément Faugeras, Marek Potemski, Aleksander Bogucki, Jan Suffczyński, Piotr Kossacki, Andrew R Wildes, Benoit Grémaud, and Andrés Saúl, "Magnetoelastic interaction in the two-dimensional magnetic material $\mathrm{MnPS}_{3}$ studied by first principles calculations and Raman experiments," 2D Mater. 7, 035030 (2020).

[12] Xingzhi Wang, Jun Cao, Zhengguang Lu, Arielle Cohen, Hikari Kitadai, Tianshu Li, Qishuo Tan, Matthew Wilson, Chun Hung Lui, Dmitry Smirnov, et al., "Spininduced linear polarization of photoluminescence in antiferromagnetic van der Waals crystals," Nat. Mater. 20, 964-970 (2021).

[13] Zhuoliang Ni, AV Haglund, H Wang, B Xu, C Bernhard, DG Mandrus, X Qian, EJ Mele, CL Kane, and Liang $\mathrm{Wu}$, "Imaging the Néel vector switching in the monolayer antiferromagnet $\mathrm{MnPSe}_{3}$ with strain-controlled Ising or- 
der," Nat. Nanotechnol. 16, 782-787 (2021).

[14] Kyle Hwangbo, Qi Zhang, Qianni Jiang, Yong Wang, Jordan Fonseca, Chong Wang, Geoffrey M Diederich, Daniel R Gamelin, Di Xiao, Jiun-Haw Chu, Wang Yao, and Xiaodong $\mathrm{Xu}$, "Highly anisotropic excitons and multiple phonon bound states in a van der Waals antiferromagnetic insulator," Nat. Nanotechnol. 16, 655-660 (2021).

[15] Dmytro Afanasiev, Jorrit R. Hortensius, Mattias Matthiesen, Samuel Mañas-Valero, Makars Siškins, Martin Lee, Edouard Lesne, Herre S. J. van der Zant, Peter G. Steeneken, Boris A. Ivanov, Eugenio Coronado, and Andrea D. Caviglia, "Controlling the anisotropy of a van der waals antiferromagnet with light," Sci. Adv. 7, eabf3096 (2021).

[16] Bevin Huang, Genevieve Clark, Efrén NavarroMoratalla, Dahlia R. Klein, Ran Cheng, Kyle L. Seyler, Ding Zhong, Emma Schmidgall, Michael A. McGuire, David H. Cobden, Wang Yao, Di Xiao, Pablo JarilloHerrero, and Xiaodong $\mathrm{Xu}$, "Layer-dependent ferromagnetism in a van der Waals crystal down to the monolayer limit," Nature 546, 270-273 (2017).

[17] Cheng Gong, Lin Li, Zhenglu Li, Huiwen Ji, Alex Stern, Yang Xia, Ting Cao, Wei Bao, Chenzhe Wang, and Yuan Wang, "Discovery of intrinsic ferromagnetism in two-dimensional van der Waals crystals," Nature $\mathbf{5 4 6}$, 265-269 (2017).

[18] Weijong Chen, Zeyuan Sun, Zhongjie Wang, Lehua Gu, Xiaodong $\mathrm{Xu}$, Shiwei $\mathrm{Wu}$, and Chunlei Gao, "Direct observation of van der Waals stacking dependent interlayer magnetism," Science 366, 983-987 (2019).

[19] Lucas Thiel, Zhe Wang, Märta A Tschudin, Dominik Rohner, Ignacio Gutiérrez-Lezama, Nicolas Ubrig, Marco Gibertini, Enrico Giannini, Alberto F Morpurgo, and Patrick Maletinsky, "Probing magnetism in 2D materials at the nanoscale with single-spin microscopy," Science 364, 973-976 (2019).

[20] Dahlia R Klein, David MacNeill, Jose L Lado, David Soriano, Efrén Navarro-Moratalla, Kenji Watanabe, Takashi Taniguchi, Soham Manni, Paul Canfield, and Joaquín Fernández-Rossier, "Probing magnetism in 2D van der Waals crystalline insulators via electron tunneling," Science 360, 1218-1222 (2018).

[21] Tiancheng Song, Xinghan Cai, Matisse Wei-Yuan Tu, Xiaoou Zhang, Bevin Huang, Nathan P Wilson, Kyle L Seyler, Lin Zhu, Takashi Taniguchi, and Kenji Watanabe, "Giant tunneling magnetoresistance in spin-filter van der Waals heterostructures," Science 360, 1214-1218 (2018).

[22] Kenneth S Burch, David Mandrus, and Je-Geun Park, "Magnetism in two-dimensional van der Waals materials," Nature 563, 47-52 (2018).

[23] Bevin Huang, Genevieve Clark, Dahlia R Klein, David MacNeill, Efrén Navarro-Moratalla, Kyle L Seyler, Nathan Wilson, Michael A McGuire, David H Cobden, Di Xiao, et al., "Electrical control of 2D magnetism in bilayer $\mathrm{CrI}_{3}$," Nature Nanotechnology 13, 544-548 (2018).

[24] Yujun Deng, Yijun Yu, Yichen Song, Jingzhao Zhang, Nai Zhou Wang, Zeyuan Sun, Yangfan Yi, Yi Zheng Wu, Shiwei Wu, Junyi Zhu, Jing Wang, Xian Hui Chen, and Yuanbo Zhang, "Gate-tunable room-temperature ferromagnetism in two-dimensional $\mathrm{Fe}_{3} \mathrm{GeTe}_{2}$," Nature 563, 94-99 (2018).

[25] Kin Fai Mak, Jie Shan, and Daniel C. Ralph, "Probing and controlling magnetic states in 2D layered magnetic materials," Nat. Rev. Phys. 1, 646-661 (2019).

[26] Cheng Gong and Xiang Zhang, "Two-dimensional magnetic crystals and emergent heterostructure devices," Science 363, 6428 (2019).

[27] M Gibertini, M Koperski, AF Morpurgo, and KS Novoselov, "Magnetic 2D materials and heterostructures," Nat. Nanotechnol. 14, 408-419 (2019).

[28] Zeyuan Sun, Yangfan Yi, Tiancheng Song, Genevieve Clark, Bevin Huang, Yuwei Shan, Shuang Wu, Di Huang, Chunlei Gao, Zhanghai Chen, Michael McGuire, Ting Cao, Di Xiao, Wei-Tao Liu, Wang Yao, Xiaodong Xu, and Shiwei Wu, "Giant nonreciprocal second-harmonic generation from antiferromagnetic bilayer $\mathrm{CrI}_{3}$," Nature 572, 497-501 (2019).

[29] Hao Chu, Chang Jae Roh, Joshua O. Island, Chen Li, Sungmin Lee, Jingjing Chen, Je-Geun Park, Andrea F. Young, Jong Seok Lee, and David Hsieh, "Linear Magnetoelectric Phase in Ultrathin $\mathrm{MnPS}_{3}$ Probed by Optical Second Harmonic Generation," Phys. Rev. Lett. 124, 027601 (2020).

[30] Amber McCreary, Jeffrey R Simpson, Thuc T Mai, Robert D McMichael, Jason E Douglas, Nicholas Butch, Cindi Dennis, Rolando Valdes Aguilar, and Angela R Hight Walker, "Quasi-two-dimensional magnon identification in antiferromagnetic $\mathrm{FePS}_{3}$ via magneto-Raman spectroscopy," Phys. Rev. B 101, 064416 (2020).

[31] Thuc T Mai, KF Garrity, Amber McCreary, J Argo, JR Simpson, V Doan-Nguyen, R Valdes Aguilar, and AR Walker, "Magnon-phonon hybridization in quasi2D antiferromagnet $\mathrm{MnPSe}_{3}$," arXiv preprint (2020), arxiv.org/abs/2011.12557.

[32] Manfred Fiebig, Dietmar Fröhlich, BB Krichevtsov, and Roman V Pisarev, "Second harmonic generation and magnetic-dipole-electric-dipole interference in antiferromagnetic $\mathrm{Cr}_{2} \mathrm{O}_{3}$," Phys. Rev. Lett. 73, 2127 (1994).

[33] Manfred Fiebig, Victor V. Pavlov, and Roman V. Pisarev, "Second-harmonic generation as a tool for studying electronic and magnetic structures of crystals: review," J. Opt. Soc. Am. B 22, 96-118 (2005).

[34] V. N. Muthukumar, R. Valentí, and C. Gros, "Microscopic model of nonreciprocal optical effects in $\mathrm{Cr}_{2} \mathrm{O}_{3}$," Phys. Rev. Lett. 75, 2766-2769 (1995).

[35] M Scagliotti, M Jouanne, M Balkanski, G Ouvrard, and G Benedek, "Raman scattering in antiferromagnetic $\mathrm{FePS}_{3}$ and $\mathrm{FePSe}_{3}$ crystals," Phys. Rev. B 35, 7097 (1987).

[36] E. Ressouche, M. Loire, V. Simonet, R. Ballou, A. Stunault, and A. Wildes, "Magnetoelectric $\mathrm{MnPS}_{3}$ as a candidate for ferrotoroidicity," Phys. Rev. B 82, 100408 (2010).

[37] HM Rønnow, AR Wildes, and ST Bramwell, "Magnetic correlations in the $2 \mathrm{D} \mathrm{S}=52$ honeycomb antiferromagnet $\mathrm{MnPS}_{3}$," Physica B: Condens. Matter 276, 676-677 (2000).

[38] AR Wildes, HM Rønnow, B Roessli, MJ Harris, and KW Godfrey, "Static and dynamic critical properties of the quasi-two-dimensional antiferromagnet $\mathrm{MnPS}_{3}$," Phys. Rev. B 74, 094422 (2006).

[39] Kiichi Okuda, Ko Kurosawa, Shozo Saito, Makoto Honda, Zhihong Yu, and Muneyuki Date, "Magnetic Properties of Layered Compound $\mathrm{MnPS}_{3}$," J. Phys. Soc. Japan 55, 4456-4463 (1986).

[40] C. Pich and F. Schwabl, "Spin-wave dynamics of two- 
dimensional isotropic dipolar honeycomb antiferromagnets," Journal of Magnetism and Magnetic Materials 148, 30 - 31 (1995).

[41] Liang $\mathrm{Wu}$, Shreyas Patankar, Takahiro Morimoto, Nityan L Nair, Eric Thewalt, Arielle Little, James G Analytis, Joel E Moore, and Joseph Orenstein, "Giant anisotropic nonlinear optical response in transition metal monopnictide Weyl semimetals," Nat. Phys. 13, 350-355 (2017).

[42] See Supplemental Material for more experimental data and derivations, which includes Ref. [43-47].

[43] Sungmin Lee, Ki-Young Choi, Sangik Lee, Bae Ho Park, and Je-Geun Park, "Tunneling transport of mono- and few-layers magnetic van der Waals $\mathrm{MnPS}_{3}$," APL Mater. 4, 086108 (2016).

[44] Lukas Mennel, Marco Furchi, Stefan Wachter, Matthias Paur, Dmitry Polyushkin, and Thomas Mueller, "Optical imaging of strain in two-dimensional crystals," Nat. Commun. 9, 516 (2018).

[45] Lukas Mennel, Matthias Paur, and Thomas Mueller, "Second harmonic generation in strained transition metal dichalcogenide monolayers: $\mathrm{MoS}_{2}, \mathrm{MoSe}_{2}, \mathrm{WS}_{2}$, and $\mathrm{WSe}_{2}, "$ APL Photonics 4, 034404 (2019).

[46] Zheng Liu, Matin Amani, Sina Najmaei, Quan Xu, Xiaolong Zou, Wu Zhou, Ting Yu, Caiyu Qiu, A Glen Birdwell, Frank J Crowne, Robert Vajtai, Boris I Yakobson, Zhenhai Xia, Madan Dubey, Ajayan Pulickel M, and Jun Lou, "Strain and structure heterogeneity in $\mathrm{MoS}_{2}$ atomic layers grown by chemical vapour deposition," Nat. Commun. 5, 5246 (2014).

[47] Qianhui Zhang, Zhenyue Chang, Guanzhong Xu, Ziyu Wang, Yupeng Zhang, Zai-Quan Xu, Shujian Chen, Qiaoliang Bao, Jefferson Zhe Liu, Yui-Wing Mai, Wenhui Duan, Michael S. Fuhrer, and Changxi Zheng, "Strain relaxation of monolayer WS2 on plastic substrate," Adv. Funct. Mater. 26, 8707-8714 (2016).

[48] A Ron, E Zoghlin, L Balents, SD Wilson, and D Hsieh, "Dimensional crossover in a layered ferromagnet detected by spin correlation driven distortions," Nat. Commun. 10, 1654 (2019).

[49] Mervin Zhao, Ziliang Ye, Ryuji Suzuki, Yu Ye, Hanyu Zhu, Jun Xiao, Yuan Wang, Yoshihiro Iwasa, and Xiang Zhang, "Atomically Phase-Matched Second-Harmonic Generation in a 2D Crystal," Light Sci. Appl. 5, e16131 (2016).

[50] Sabine N. Neal, Heung-Sik Kim, Kevin A. Smith, Amanda V. Haglund, David G. Mandrus, Hans A. Bechtel, G. Lawrence Carr, Kristjan Haule, David Vanderbilt, and Janice L. Musfeldt, "Near-field infrared spectroscopy of monolayer $\mathrm{MnPS}_{3}$," Phys. Rev. B 100, 075428 (2019).

[51] S. N. Neal, H.-S. Kim, K. R. O'Neal, A. V. Haglund, K. A. Smith, D. G. Mandrus, H. A. Bechtel, G. L. Carr, K. Haule, David Vanderbilt, and J. L. Musfeldt, "Symmetry crossover in layered $\mathrm{MPS}_{3}$ complexes $(M=\mathrm{Mn}, \mathrm{Fe}, \mathrm{Ni})$ via near-field infrared spectroscopy," Phys. Rev. B 102, 085408 (2020). 


\title{
Supplementary Material for
}

\section{Direct imaging of antiferromagnetic domains and anomalous layer-dependent mirror symmetry breaking in atomically thin $\mathrm{MnPS}_{3}$}

\author{
Zhuoliang Ni, ${ }^{1}$ Huiqin Zhang, ${ }^{2}$ David Hopper, ${ }^{2,1}$ Amanda V. \\ Haglund, ${ }^{3}$ Nan Huang, ${ }^{3}$ Deep Jariwala, ${ }^{2}$ Lee Bassett, ${ }^{2}$ David G. \\ Mandrus, ${ }^{3,4}$ Eugene J. Mele, ${ }^{1}$ Charles L. Kane, ${ }^{1}$ and Liang $\mathrm{Wu}^{1, *}$ \\ ${ }^{1}$ Department of Physics and Astronomy, University of Pennsylvania, \\ Philadelphia, Pennsylvania 19104, U.S.A \\ ${ }^{2}$ Department of Electrical and System Engineering, \\ University of Pennsylvania, Philadelphia, Pennsylvania 19104, U.S.A \\ ${ }^{3}$ Department of Materials Science and Engineering, \\ University of Tennessee, Knoxville, TN 37996, U.S.A. \\ ${ }^{4}$ Materials Science and Technology Division, \\ Oak Ridge National Laboratory, Oak Ridge, TN, 37831, U.S.A.
}

\footnotetext{
* liangwu@sas.upenn.edu
} 


\section{Note S1. Symmetry information of the polarization-resolved SHG intensity}

In this study, we mainly focus on two second-harmonic contributions in $\mathrm{MnPS}_{3}$ : the spin-induced electric dipole $(\mathrm{ED})$ term $\left(\chi_{i j k}^{E D} E_{i} E_{j}\right)$ and electric quadruple (EQ) term $\left(\chi_{i j k l}^{E Q} E_{j} \nabla_{k} E_{l}\right)$. The rank 3 tensor $\chi_{i j k}^{E D}$ and rank 4 tensor $\chi_{i j k}^{E D}$ follow the symmetry of the $\mathrm{MnPS}_{3}$ crystal. Here, we only consider the case that the mirror symmetry of $\mathrm{MnPS}_{3}$ is present.

\section{A. $T>T_{N}$}

Above Néel temperature, the point group of $\mathrm{MnPS}_{3}$ crystal is $2 / \mathrm{m}$. The $\chi_{i j k}^{E D} E_{i} E_{j}$ is zero because of the presence of the inversion symmetry. Under normal incidence, we can simplify the EQ term as $\chi_{i j z l}^{E Q} k_{z} E_{j} E_{l}(j, l=x, y)$. According to the mirror symmetry perpendicular to the b-axis we have $\chi_{x x z y}^{E Q}=\chi_{x y z x}^{E Q}=\chi_{y x z x}^{E Q}=\chi_{y y z y}^{E Q}=0$. In conclusion, the SHG intensity for parallel and crossed configuration should be

$$
I_{\text {parallel }}\left(2 \omega, T>T_{N}\right) \propto\left(\left(\chi_{x y z y}^{E Q}+2 \chi_{y x z y}^{E Q}\right) \sin ^{2} \phi \cos \phi+\chi_{x x z x}^{E Q} \cos ^{3} \phi\right)^{2} I(\omega)^{2}
$$

and

$$
I_{\text {crossed }}\left(2 \omega, T>T_{N}\right) \propto\left(\left(-\chi_{x x z x}^{E Q}+2 \chi_{y x z y}^{E Q}\right) \sin \phi \cos ^{2} \phi-\chi_{x y z y}^{E Q} \sin ^{3} \phi\right)^{2} I(\omega)^{2},
$$

where $I(\omega)$ is the incident power and $\phi$ is the angle between a-axis and polarization of incident light. When $\phi=\pi / 2, I_{\text {parallel }}(2 \omega)=0$, and when $\phi=0, I_{\text {crossed }}(2 \omega)=0$, indicating no SHG output is from the b-axis.

\section{B. $T<T_{N}$}

When the long-range antiferromagnetic order forms, the $\mathrm{MnPS}_{3}$ breaks inversion symmetry, so the $\chi^{E D}$ is non-zero. According to its magnetic point group $2 \% / \mathrm{m}$, we have $\chi_{x x y}^{E D}=\chi_{x y x}^{E D}=\chi_{y x x}^{E D}=\chi_{y y y}^{E D}=0$. Then the second-harmonic electric field for the parallel and crossed configurations from ED term are,

$$
E_{\text {parallel }}^{E D}\left(2 \omega, T<T_{N}\right) \propto\left(\left(\chi_{x y y}^{E D}+2 \chi_{y x y}^{E D}\right) \sin ^{2} \phi \cos \phi+\chi_{x x x}^{E D} \cos ^{3} \phi\right) I(\omega)
$$



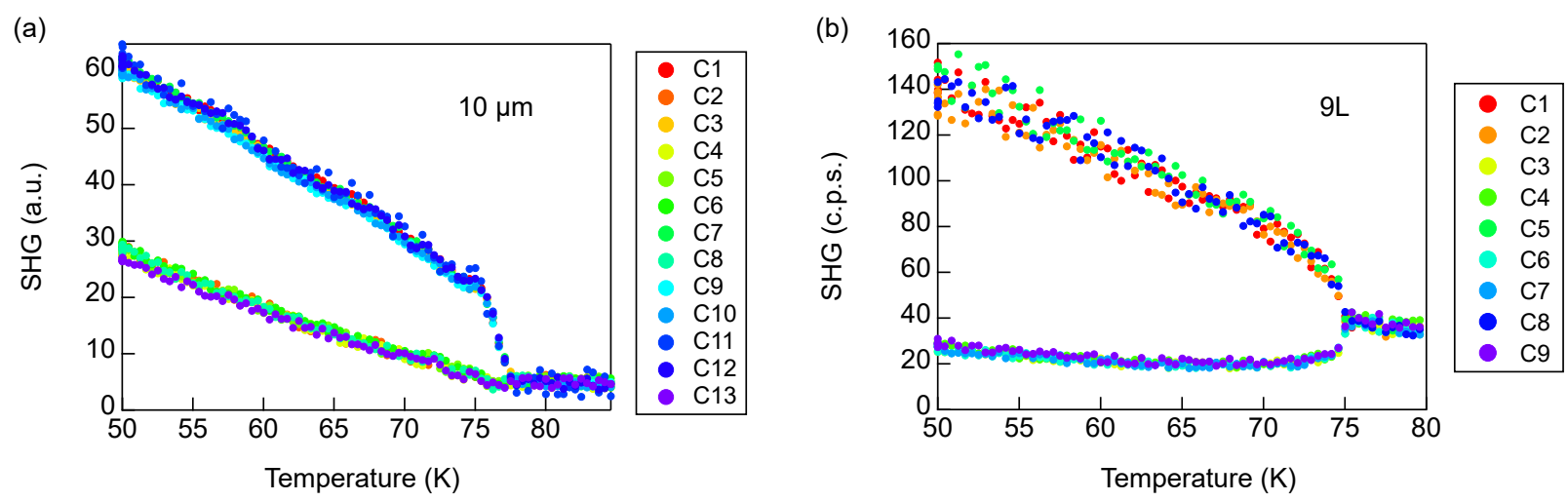

Figure S1. Consecutive temperature dependence of SHG measurement on $\mathrm{MnPS}_{3}$. (a) Cooling curves under 13 consecutive thermal cycles on the $10 \mu \mathrm{m}$ bulk crystal shown in Fig. 1 in the main text. (b) Cooling curves under 9 consecutive thermal cycles on the 9L sample shown in Fig.2 in the main text. In both cases, only two kinds of SHG signals are observed.

and

$$
E_{\text {crossed }}^{E D}\left(2 \omega, T<T_{N}\right) \propto\left(\left(-\chi_{x x x}^{E D}+2 \chi_{y x y}^{E D}\right) \sin \phi \cos ^{2} \phi-\chi_{x y y}^{E D} \sin ^{3} \phi\right) I(\omega) .
$$

The symmetry of the tensor $\chi_{i j k l}^{E Q}$ is identical to the case of $T<T_{n}$, so

$$
E_{\text {parallel }}^{E Q}\left(2 \omega, T<T_{N}\right) \propto\left(\left(\chi_{x y z y}^{E Q}+2 \chi_{y x z y}^{E Q}\right) \sin ^{2} \phi \cos \phi+\chi_{x x z x}^{E Q} \cos ^{3} \phi\right) I(\omega)
$$

and

$$
E_{\text {crossed }}^{E Q}\left(2 \omega, T<T_{N}\right) \propto\left(\left(-\chi_{x x z x}^{E Q}+2 \chi_{y x z y}^{E Q}\right) \sin \phi \cos ^{2} \phi-\chi_{x y z y}^{E Q} \sin ^{3} \phi\right) I(\omega) .
$$

Finally, the SHG intensity below $T_{N}$ is

$$
I_{\text {parallel }}^{\text {total }}\left(2 \omega, T<T_{N}\right) \propto\left( \pm E_{\text {parallel }}^{E D}\left(2 \omega, T<T_{N}\right)+E_{\text {parallel }}^{E Q}\left(2 \omega, T<T_{N}\right)\right)^{2}
$$

and

$$
I_{\text {crossed }}^{\text {total }}\left(2 \omega, T<T_{N}\right) \propto\left( \pm E_{\text {crossed }}^{E D}\left(2 \omega, T<T_{N}\right)+E_{\text {crossed }}^{E Q}\left(2 \omega, T<T_{N}\right)\right)^{2},
$$

where \pm corresponds to two different domains.

\section{Note S2. SHG measurements on $\mathrm{NiPS}_{3}$}

Here we measure a centrosymmetric van der Waals antiferromagnetic material $\mathrm{NiPS}_{3}$. The $\mathrm{NiPS}_{3}$ crystal has the same space and point group in the paramagnetic phase as $\mathrm{MnPS}_{3}$, 

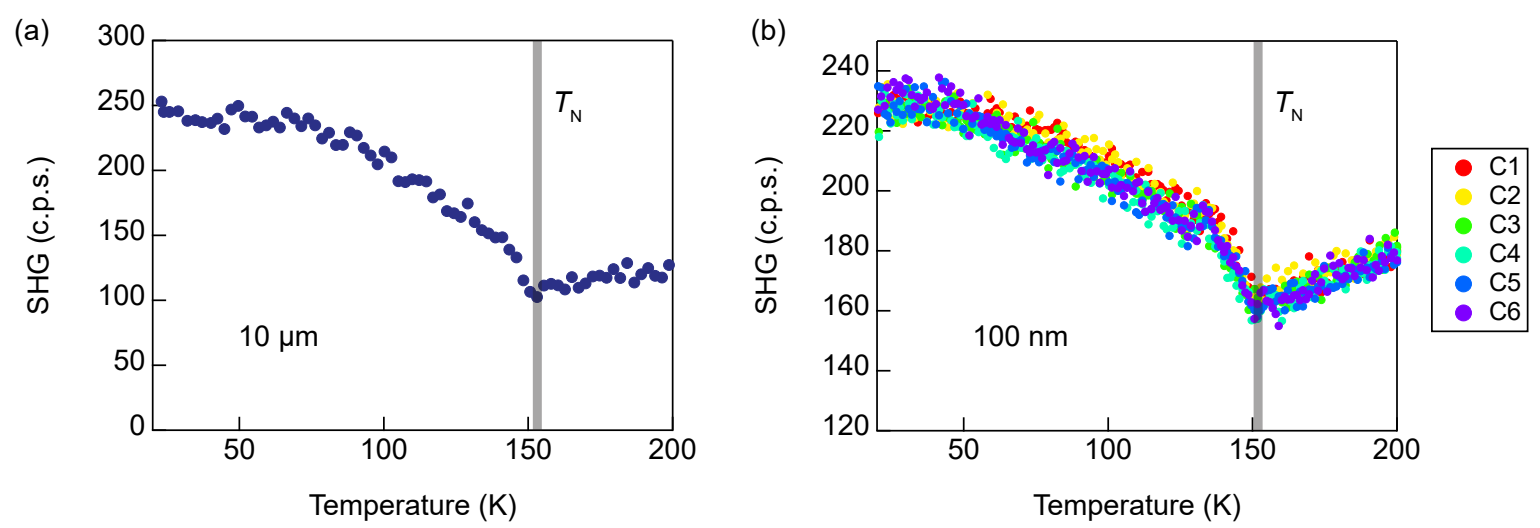

Figure S2. Temperature dependence of SHG intensity of NiPS $_{3}$. (a) Temperature dependence of SHG intensity of a $\mathrm{NiPS}_{3}$ bulk crystal of the thickness around $10 \mu \mathrm{m}$. A transition-like feature happens around $153 \mathrm{~K}$, which is the antiferromagnetic transition temperature of $\mathrm{NiPS}_{3}$. (b) Temperature dependence of SHG intensity of a thick $\mathrm{NiPS}_{3}$ flake exfoliated on $\mathrm{SiO}_{2} / \mathrm{Si}$ substrate. Six consecutive thermal cycles are performed to confirm the existence of only time-reversal invarient contribution.

but it is in a so-called "zigzag" AFM state under the transition temperature around $153 \mathrm{~K}$. The zigzag AFM does not break inversion, so the spin-induced c-type SHG is not allowed in this compound. However, the i-type electric quadrupole SHG is allowed and influenced by the magneto-elastic coupling with the formation of the antiferromagnetic order. The temperature dependence of a $\mathrm{NiPS}_{3}$ crystal with a thickness around $10 \mu \mathrm{m}$ is shown in Figure S2(a). Power of $5 \mathrm{~mW}$ and a beam spot diameter of $10 \mu \mathrm{m}$ are used. Note that a clear transition happens at the Néel temperature. We also measure a 100-nm thick $\mathrm{NiPS}_{3}$ flake exfoliated on the $\mathrm{SiO}_{2} / \mathrm{Si}$ wafer, and see a similar transition at around $153 \mathrm{~K}$ (Fig. S2(b)). Consecutive thermal cycles are performed to confirm that only one possible SHG signal exists below the Néel temperature, which is consistent with the absence of the spininduced c-type SHG contribution. Note that SHG was reported without a change across the the Néel temperature in $\mathrm{NiPS}_{3}$ [1]. The observation of the phase transition due to magneto-elastic coupling also shows the higher sensitivity of our setup. 


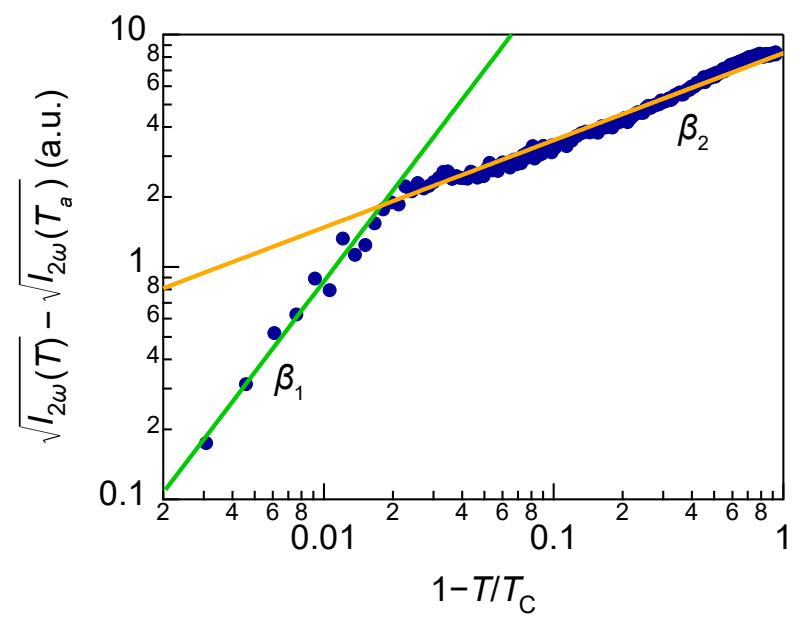

Figure S3. Temperature dependence of the second-harmonic electric field below $T_{N}$ in the log-log plot. The data (dark blue circles) are from the domain 1 of the $10 \mu \mathrm{m}$-thick $\mathrm{MnPS}_{3}$ crystal in Fig. 1 in the main text. A crossover of slope $\beta$ is observed at around $76 \mathrm{~K}$. Linear fitting is performed above and below $76 \mathrm{~K}$, as shown by the green and the yellow lines.

\section{Note S3. Crossover of the exponent component $\beta$ in $\mathrm{MnPS}_{3}$ bulk}

Near the Néel temperature, the electric-dipole second-order susceptibility $\chi^{E D}$ is proportional to $\left(1-T / T_{N}\right)^{\beta}$. In Fig.1 in the main text, we report a change of slope around $76 \mathrm{~K}$ in the temperature-dependent $\mathrm{SHG}$ in a bulk $\mathrm{MnPS}_{3}$ crystal. To study the crossover-like feature, we plot the square root of SHG signal of domain 1 (the nonzero value at $T_{N}$ is subtracted) as a function of $1-T / T_{N}$ in the log-log plot (Fig. S3). The slope of the curve is then representing the critical exponent $\beta$. A clear crossover of the slope is observed in the plot with $\beta_{1}=1.3(0)$ and $\beta_{2}=0.37(5)$. A neutron scattering measurement in $\mathrm{MnPS}_{3}$ also shows a crossover feature on the exponent coefficients at a similar temperature but with different values of $\beta$ [2], where the critical exponents are more accurate as it measures the staggered magnetization. Note that $\chi_{i j k}^{E D}(T) \propto M \propto\left(T_{N}-T\right)^{\beta}$ [3]. If the EQ SHG does not depend on temperature below $T_{N}$, then we will see similar critical exponents as neutrom scattering. Therefore, we conclude that electric-quadruple contribution is also dependent on temperature and can show a phase-transition-like behavior ( $\mathrm{See} \mathrm{NiPS}_{3}$ data in Note $\mathrm{S} 2$ ). In this regard, the $\beta_{1}$ and $\beta_{2}$ in Fig. $\mathbf{S 3}$ are not purely related with $\chi^{E D}$ as it also include the EQ SHG that is also dependent on the temperature below $T_{N}$. 


\section{Note S4. Layer characterization of few-layer $\mathrm{MnPS}_{3}$}

Typical optical images of different layers are shown in Fig. S4(a). We use two methods to distinguish the number of layers, including atomic force microscopy and optical contrast. For the atomic force microscopy measurement, the height information of the contact mode is used. A single-layer step of around $0.73 \mathrm{~nm}$ can be seen from Fig. S4(e). The height of the monolayer is measured to be around $1.3 \mathrm{~nm}$, which is larger than a single-layer step. It is possible because of the existence of an air layer on the sample surface. Layer-dependent sample thickness is shown in Fig. $\mathbf{S 4}(\mathrm{a})$ by open red circles, and a linear fit is marked by the black line. We also use optical contrast to determine the layer numbers. We extract the red channel of optical image and calculate the optical contrast for different samples by

$\eta=\frac{I_{\text {sample }}-I_{\text {substrate }}}{I_{\text {sample }}+I_{\text {substrate }}}$. The layer-dependent optical contrast is plotted in Fig. S4(b) by open red circles with a linear fit marked by a black line.

\section{Note S5. Extra data for the bilayer $\mathrm{MnPS}_{3}$ sample.}

Here we show some extra data of the bilayer sample in the main text. The optical image, atomic force microscopy mapping, and SHG intensity mapping at $5 \mathrm{~K}$ and $65 \mathrm{~K}$ are shown in Fig. S5. Clearly, the SHG signal exists at $5 \mathrm{~K}$ while almost disappears at $65 \mathrm{~K}$ for the left-side bilayer area. The data shown in the main text was measured at the same spot in the middle of the left-side bilayer area.

To exclude the possibility of degradation of the bilayer sample during the sample preparation process, we also measured a bilayer sample $\mathrm{S} 2$ exfoliated in a glove box with the $\mathrm{O}_{2}$ and water level less than 1 ppm. The data are shown in Fig. S6. The transition temperature extracted from Fig. S6 (b) is $61 \mathrm{~K}$, and the SHG intensity is around 1.5 c.p.s.. These values are consistent with those from the bilayer sample exfoliated in air shown in the main text.

\section{Note S6. Characterization of monolayer $\mathrm{MnPS}_{3}$ samples.}

We measured the data from three different monolayer $\mathrm{MnPS}_{3}$ samples, and did not observe a temperature-dependent SHG within the sensitivity of our setup. The data of the monolayer shown in the main text were measured under the laser power of $2.0 \mathrm{~mW}$. The monolayer 
(a)

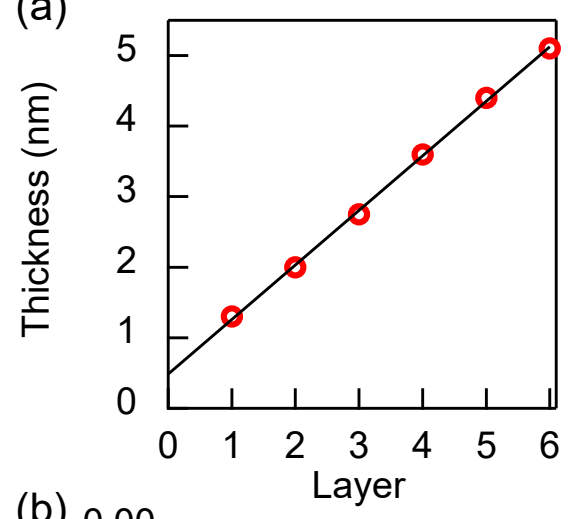

(b)

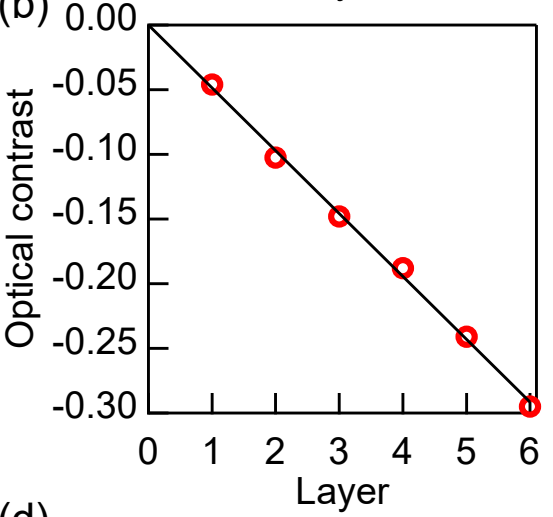

(d)

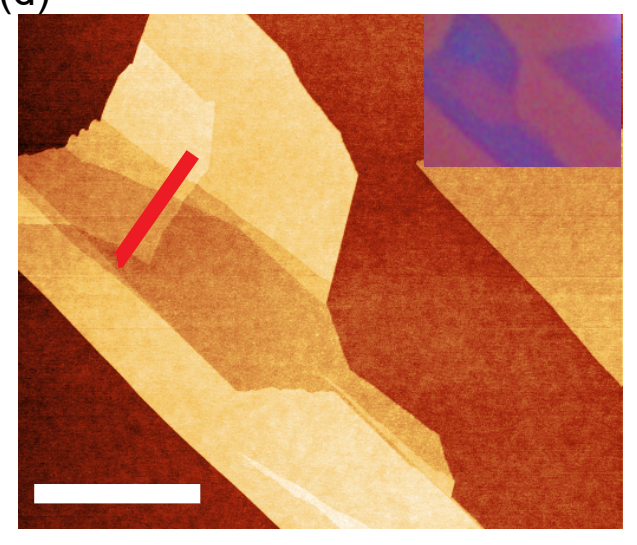

(c)

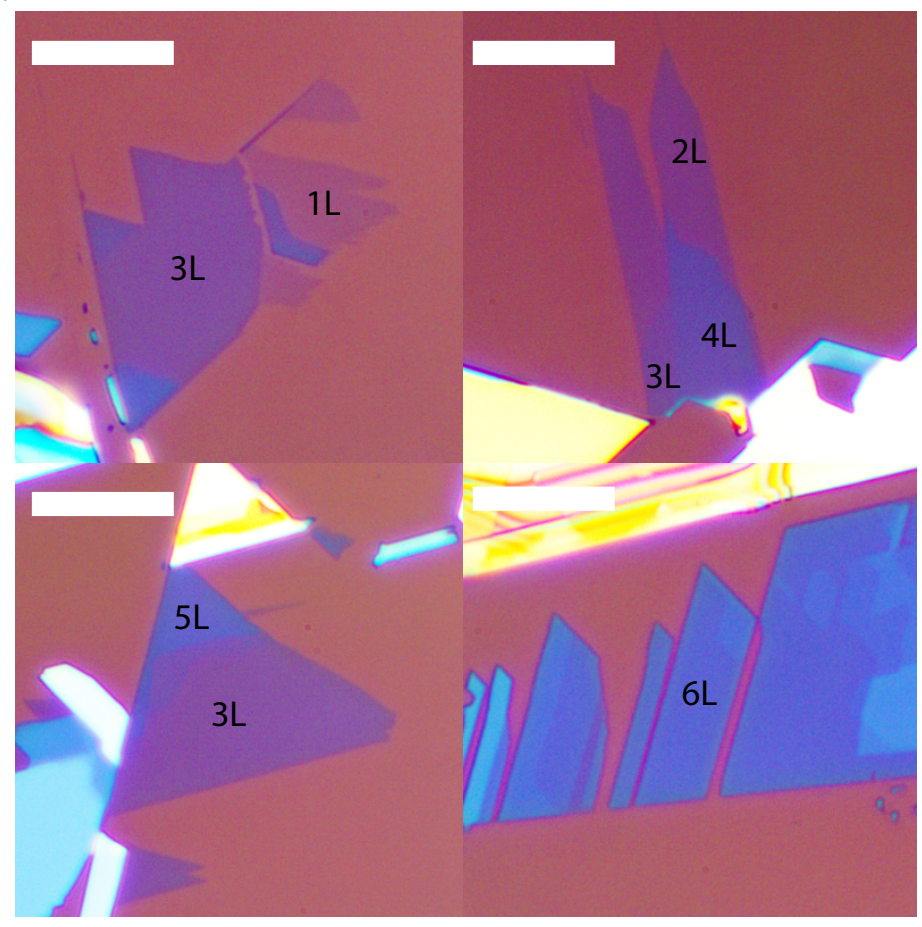

(e)

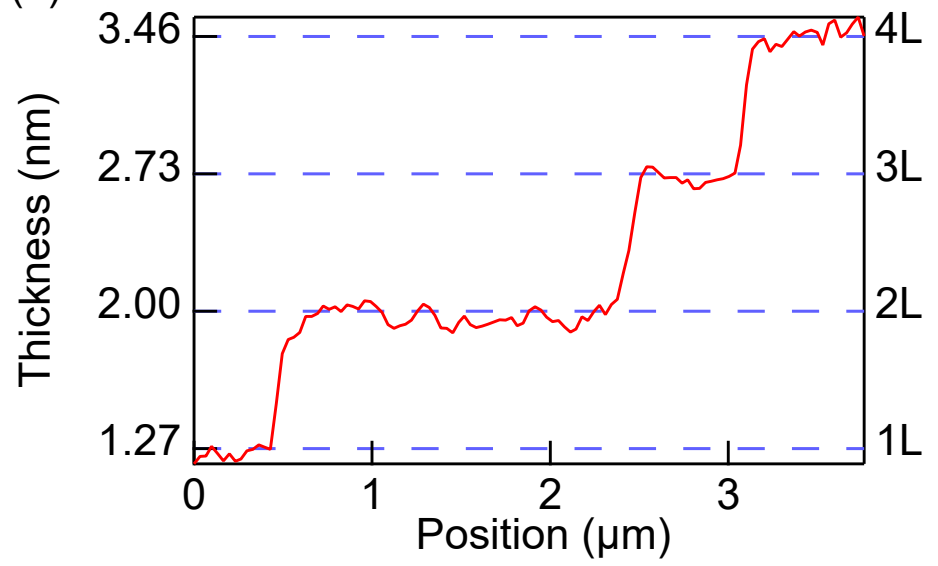

Figure S4. Determination of layer numbers. (a) Layer-dependent thickness measured by atomic force microscopy. (b) Layer-dependent optical contrast measured determined by the red channel of the optical image. (c) Optical images of typical few-layer $\mathrm{MnPS}_{3}$. Scale bar: $20 \mu \mathrm{m}$. The white balance is adjusted from the default setting to maximize the contrast of different layers. (d) Atomic force microscopy image of a multi-layer flake. (Inset: the optical image of the same flake). Scale bar: $5 \mu \mathrm{m}$. (e) A line cut of the red line shown in (d) with a single layer step of around $0.73 \mathrm{~nm}$. 
(a)

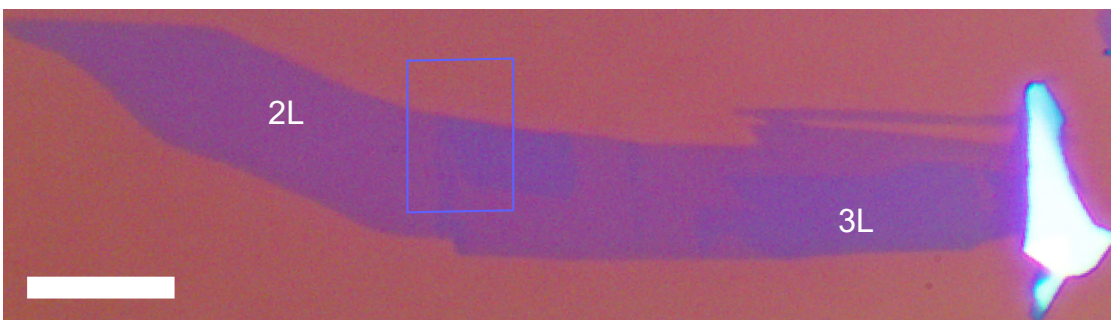

(b)

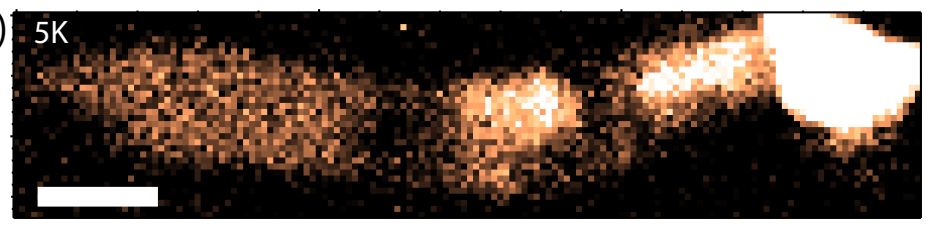

(c) $65 \mathrm{~K}$

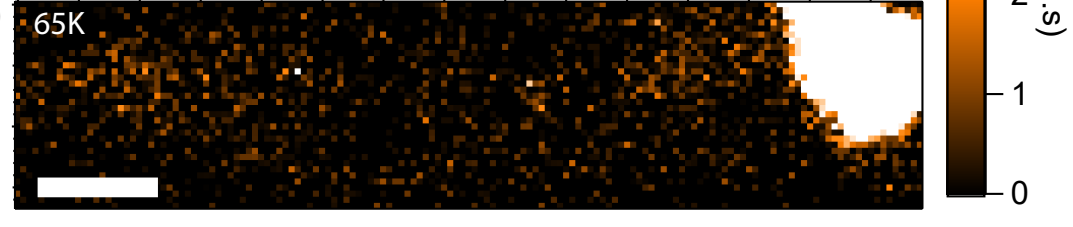

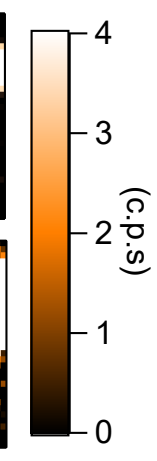

(d)

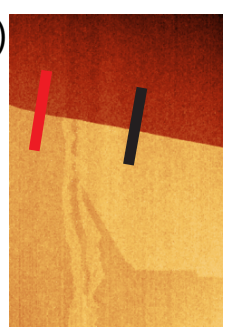

(e) 3.0

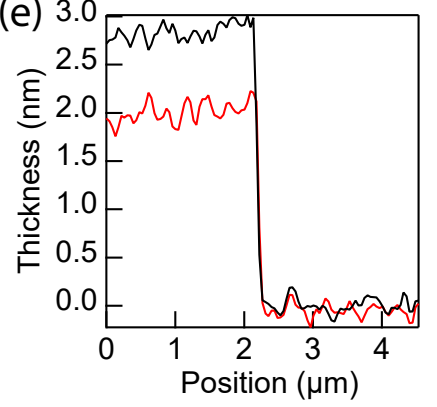

Figure S5. Extra data for the bilayer $\operatorname{MnPS}_{3}$ sample in the main text. (a) Optical image of the sample. Scale bar: $20 \mu \mathrm{m}$. (b) SHG intensity mapping at $5 \mathrm{~K}$. Scale bar: $20 \mu \mathrm{m}$. (c) SHG intensity mapping measured at $65 \mathrm{~K}$. Scale bar: $20 \mu \mathrm{m}$. (d) Atomic force microscopy height image of the boxed area in (a). The red and black cut lines are shown in (e) with a thickness step of 2.0 $\mathrm{nm}$ and $2.7 \mathrm{~nm}$ from the substrate, corresponding to a bilayer and a trilayer respectively.

sample was exfoliated in air, and the optical images are shown in Fig.S4(c). A second sample prepared and measured under the same condition shows the same temperature-independent signal. We also tried one monolayer sample exfoliated in the glove box to avoid any possible degradation during the sample preparation process, though the $\mathrm{MnPS}_{3}$ monolayer is reported to be stable in air [4]. The details of this sample are shown in Fig. S7. In this sample, we used a relatively high laser power of $5.0 \mathrm{~mW}$, which generates enough photons to measure polarization-dependent SHG patterns. We observed a small SHG signal on top of the signal from the substrate, but we did not observe any temperature-dependent signals between $5 \mathrm{~K}$ and $80 \mathrm{~K}$.

The long-range order in bulk $\mathrm{MnPS}_{3}$ is believed to result from the anisotropy and the weak interplanar coupling $[2,5]$. Except for the ordering near the 3D Néel temperature, the spin dynamics of a bulk sample are best described by the 2D Heisenberg Hamiltonian with weak XY anisotropy and interlayer coupling [2]. If atomically thin samples have the same Hamiltonian as the bulk, the absence of the long-rage order in the monolayer is either con- 
(a)

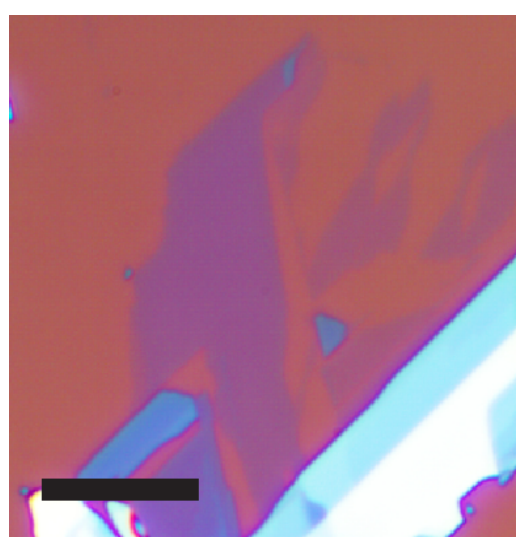

(c)

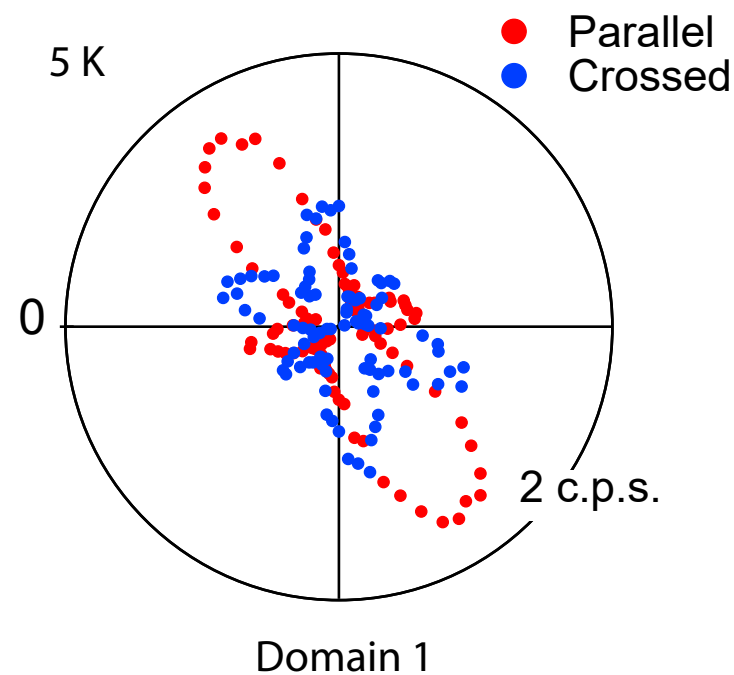

(b)

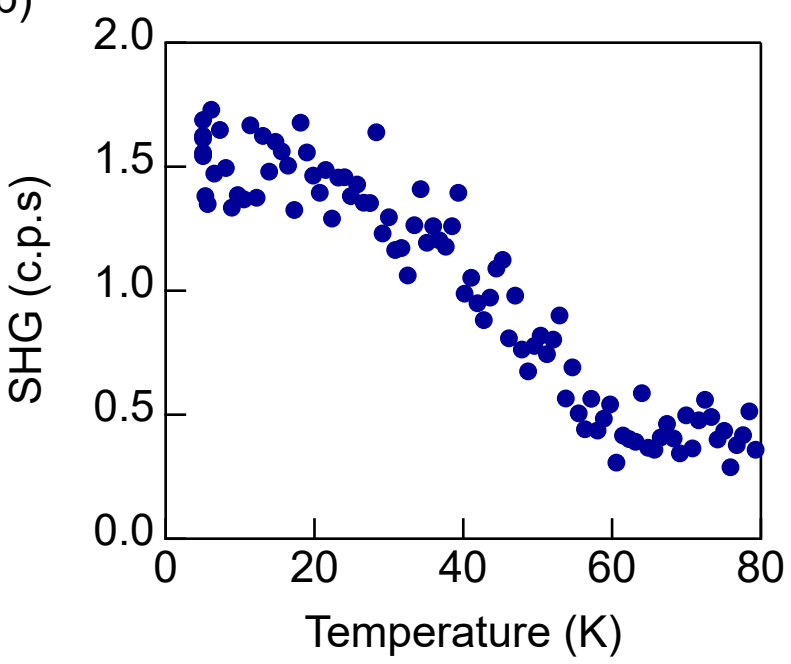

(d)

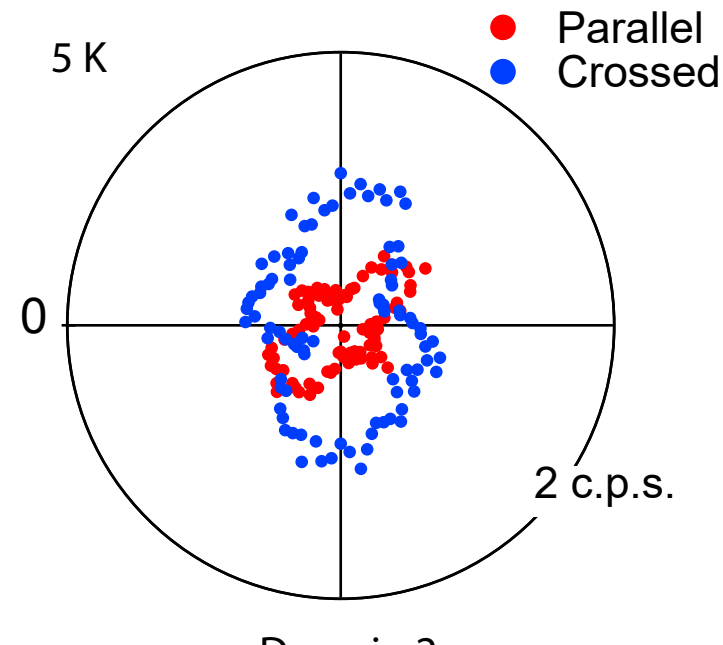

Domain 2

Figure S6. SHG data for a bilayer sample exfoliated in the glove box. (a) Optical image of the sample. Scale bar: $20 \mu \mathrm{m}$. (b) SHG intensity as a function of temperature (c-d) Polarization dependent SHG patterns in domain 1 and domain 2.

sistent with the 2D Heisenberg model without interlayer coupling or in the same universality class as the 2D XY model, which we do not have direct evidences for. Nevertheless, as we wrote in the main text, both the lattice and the spin structure of samples thinner than 10L are found to be different from the bulk, so we actually do not know the spin Hamiltonian and anisotropy in atomically thin samples. Note that the suppression of $T_{N}$ is also most prominent below 10L. It is possible that the anisotropy is strongly reduced in atomically thin limit, especially in the monolayer $[6,7]$. Also, we can not rule out the possibility of disorder 
(a)

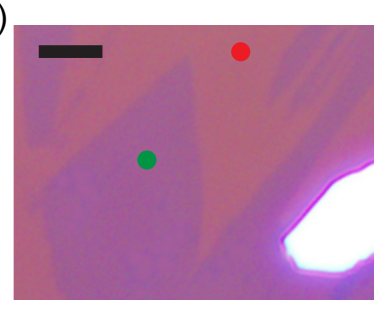

(b)

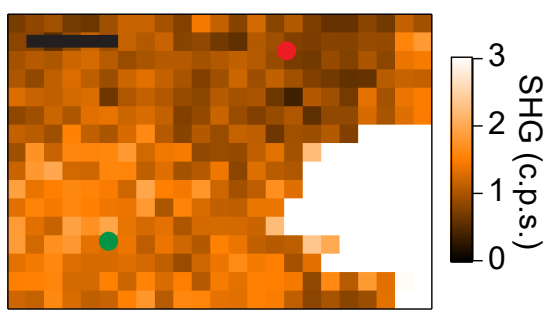

(f)

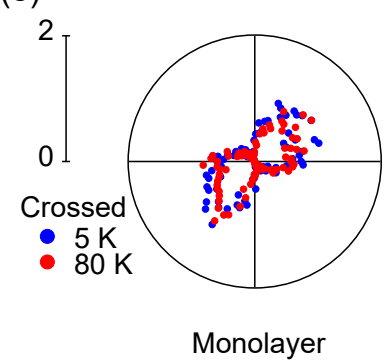

(e) (c)

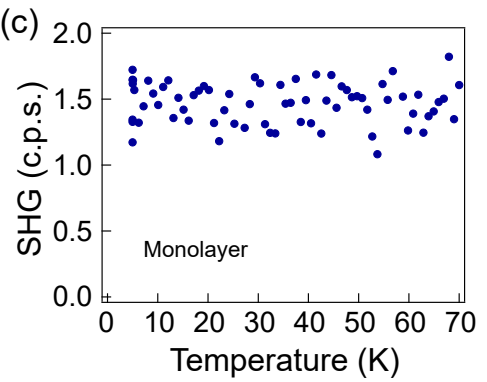

(d) unit: c.p.s.

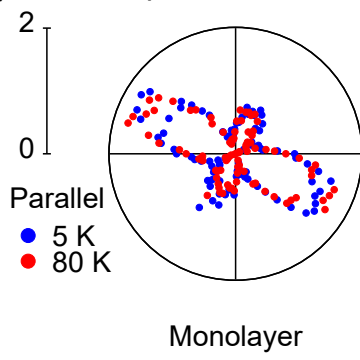

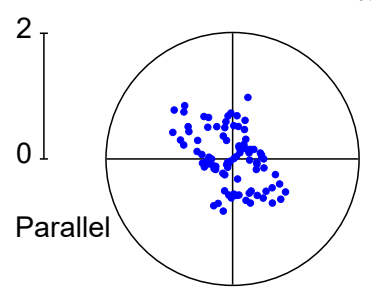

Substrate (g)

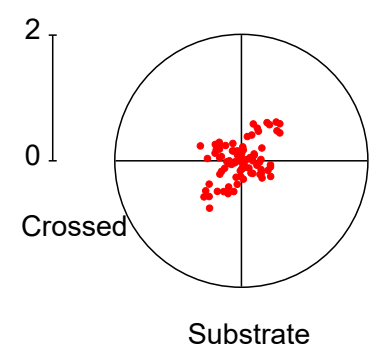

Figure S7. SHG data of the monolayer $\mathrm{MnPS}_{3}$ exfoliated in a glove box. (a) Optical image of the monolayer sample. Scale bar: $10 \mu \mathrm{m}$. (b) A SHG mapping of the monolayer sample. The region around the green dot is the monolayer and the region around the red dot is the $\mathrm{SiO}_{2} / \mathrm{Si}$ substrate. Scale bar: $10 \mu \mathrm{m}$. (c) Temperature-dependent SHG signal. (d-e) Parallel (d) and crossed (e) patterns of the monolayer sample at $5 \mathrm{~K}$ and $80 \mathrm{~K}$. (f-g) Parallel (f) and crossed (g) pattern of the substrate.

effect even though we do not see buckling from atomic force microscopy measurements. We hope our work will motivate more works on atomically thin $\mathrm{MnPS}_{3}$ especially the monolayer in the future.

\section{Note S7. Strain-tuning measurement of few-layer $\mathrm{MnPS}_{3}$ samples}

From the SHG measurement, we have confirmed that the mirror symmetry was broken below and above the Néel temperature in few-layer samples with thickless less than 11L. It is reported previously in a $7 \mathrm{~L} \mathrm{MnPS}_{3}$ sample below Néel temperature by a similar SHG measurement, which they attribute to the substrate-induced strain[1]. According to our measurement, we argue that the substrate-induced strain is not the key reason for the mirrorsymmetry breaking. First, strain modification on SHG patterns is usually quite small. The polarization-dependent SHG patterns of the few-layer samples shown in the main text are 


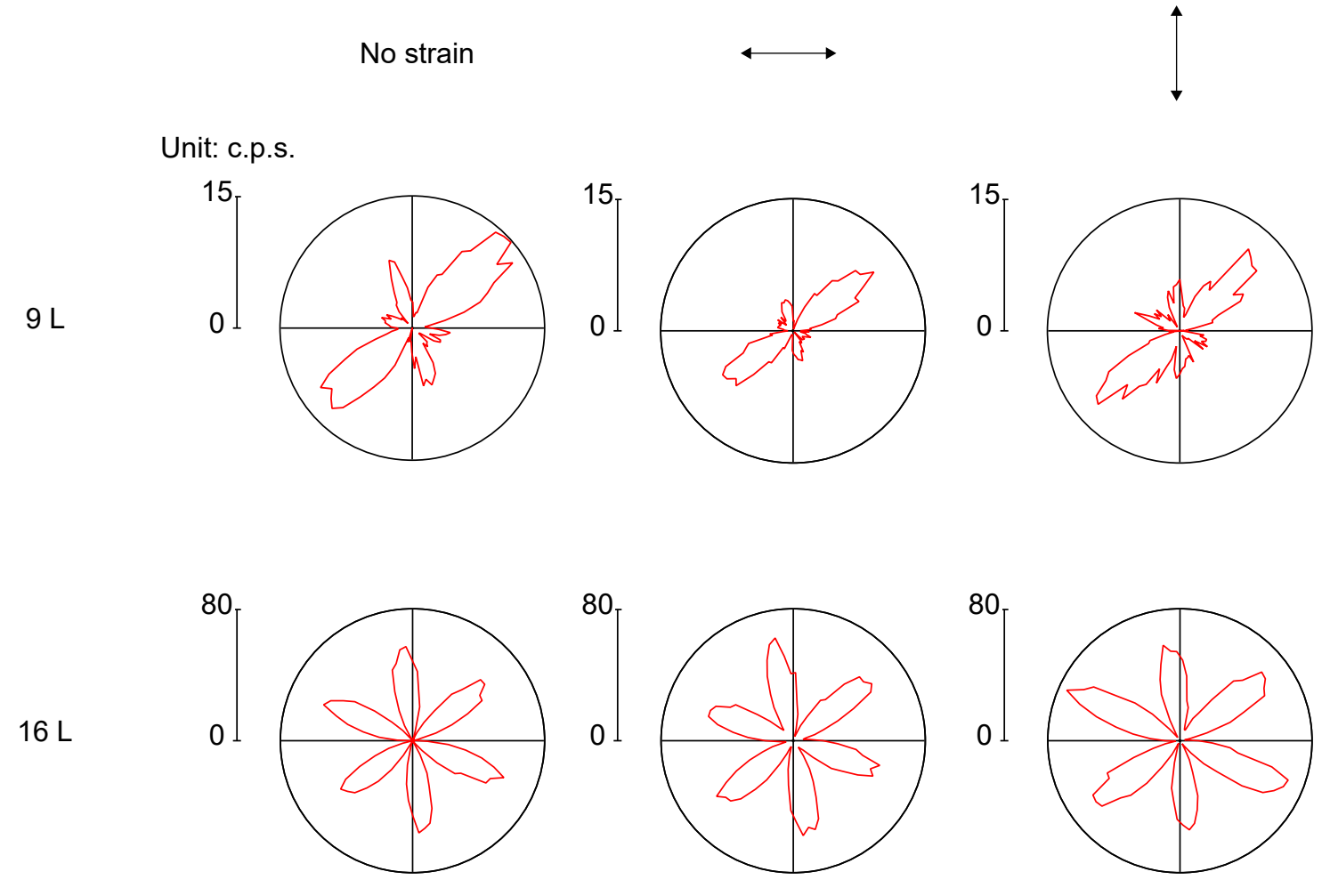

Figure S8. Crossed SHG patterns of samples on a substrate under uniaxial strain.

quite different from the six-fold patterns in the thicker samples. While the previous study on strain-dependent SHG of other 2D materials never shows such a large modification even by adding a large strain deliberately $[8,9]$.

To further justify our statement, we did a strain-tuning experiment on both thick and thin $\mathrm{MnPS}_{3}$ samples. $\mathrm{MnPS}_{3}$ were exfoliated on a polydimethylsiloxane (PDMS) substrate. The flexibility of the PDMS film enabled us to add strains on the samples in any direction[10-12]. We used a micro-mechanical stage to apply around 5\% uniaxial strain on the PDMS, which is then attached to a gold-coated wafer. The transfer ratio of the strain is reported to be around 13-15\% [10-12]. The adherence between the gold coating and PDMS was strong enough to sustain the strain at $5 \mathrm{~K}$. Fig.S8 shows the results of a 9L sample and a 16L sample measured at $5 \mathrm{~K}$ and under different strains applied on the substrates. We did not observe a clear difference between different strains in both samples. Specifically, no obvious mirror symmetry breaking was induced in the $16 \mathrm{~L}$ sample. 
[1] Hao Chu, Chang Jae Roh, Joshua O. Island, Chen Li, Sungmin Lee, Jingjing Chen, Je-Geun Park, Andrea F. Young, Jong Seok Lee, and David Hsieh, "Linear Magnetoelectric Phase in Ultrathin $\mathrm{MnPS}_{3}$ Probed by Optical Second Harmonic Generation," Phys. Rev. Lett. 124, $027601(2020)$.

[2] AR Wildes, HM Rønnow, B Roessli, MJ Harris, and KW Godfrey, "Static and dynamic critical properties of the quasi-two-dimensional antiferromagnet $\mathrm{MnPS}_{3}$," Phys. Rev. B 74, $094422(2006)$.

[3] V. N. Muthukumar, R. Valentí, and C. Gros, "Microscopic model of nonreciprocal optical effects in $\mathrm{Cr}_{2} \mathrm{O}_{3}$," Phys. Rev. Lett. 75, 2766-2769 (1995).

[4] Sungmin Lee, Ki-Young Choi, Sangik Lee, Bae Ho Park, and Je-Geun Park, "Tunneling transport of mono- and few-layers magnetic van der Waals $\mathrm{MnPS}_{3}$," APL Mater. 4, 086108 (2016).

[5] HM Rønnow, AR Wildes, and ST Bramwell, "Magnetic correlations in the 2D S=52 honeycomb antiferromagnet $\mathrm{MnPS}_{3}$," Physica B: Condens. Matter 276, 676-677 (2000).

[6] Cheng Gong, Lin Li, Zhenglu Li, Huiwen Ji, Alex Stern, Yang Xia, Ting Cao, Wei Bao, Chenzhe Wang, and Yuan Wang, "Discovery of intrinsic ferromagnetism in two-dimensional van der Waals crystals," Nature 546, 265-269 (2017).

[7] Yujun Deng, Yijun Yu, Yichen Song, Jingzhao Zhang, Nai Zhou Wang, Zeyuan Sun, Yangfan Yi, Yi Zheng Wu, Shiwei Wu, Junyi Zhu, Jing Wang, Xian Hui Chen, and Yuanbo Zhang, "Gate-tunable room-temperature ferromagnetism in two-dimensional $\mathrm{Fe}_{3} \mathrm{GeTe}_{2}$," Nature $\mathbf{5 6 3}$, 94-99 (2018).

[8] Lukas Mennel, Marco Furchi, Stefan Wachter, Matthias Paur, Dmitry Polyushkin, and Thomas Mueller, "Optical imaging of strain in two-dimensional crystals," Nat. Commun. 9, $516(2018)$.

[9] Lukas Mennel, Matthias Paur, and Thomas Mueller, "Second harmonic generation in strained transition metal dichalcogenide monolayers: $\mathrm{MoS}_{2}, \mathrm{MoSe}_{2}, \mathrm{WS}_{2}$, and $\mathrm{WSe}_{2}, " \mathrm{APL}$ Photonics 4, 034404 (2019).

[10] Zheng Liu, Matin Amani, Sina Najmaei, Quan Xu, Xiaolong Zou, Wu Zhou, Ting Yu, Caiyu Qiu, A Glen Birdwell, Frank J Crowne, Robert Vajtai, Boris I Yakobson, Zhenhai Xia, Madan 
Dubey, Ajayan Pulickel M, and Jun Lou, "Strain and structure heterogeneity in $\mathrm{MoS}_{2}$ atomic layers grown by chemical vapour deposition," Nat. Commun. 5, 5246 (2014).

[11] Qianhui Zhang, Zhenyue Chang, Guanzhong Xu, Ziyu Wang, Yupeng Zhang, Zai-Quan Xu, Shujian Chen, Qiaoliang Bao, Jefferson Zhe Liu, Yui-Wing Mai, Wenhui Duan, Michael S. Fuhrer, and Changxi Zheng, "Strain relaxation of monolayer WS2 on plastic substrate," Adv. Funct. Mater. 26, 8707-8714 (2016).

[12] Zhuoliang Ni, AV Haglund, H Wang, B Xu, C Bernhard, DG Mandrus, X Qian, EJ Mele, CL Kane, and Liang Wu, "Imaging the Néel vector switching in the monolayer antiferromagnet $\mathrm{MnPSe}_{3}$ with strain-controlled Ising order," Nat. Nanotechnol. 16, 782-787 (2021). 